\title{
PRONÓSTICO DEL ÍNDICE BURSÁTIL ECUATORIANO (ECUINDEX) MEDIANTE REDES NEURONALES
} AUTORREGRESIVAS

\section{FORECAST OF ECUADORIAN STOCK INDEX (ECUINDEX) USING AUTOREGRESSIVE NEURAL NETWORKS}

Álex Dávila ${ }^{1}$

Napoleón Sanchez-Choez ${ }^{2}$

José Luis Román-Vásquez ${ }^{3}$

1. Docente - Investigador de la Escuela Politécnica Nacional (Ecuador), Ingeniero Electrónico, MBA. E-mail: alex.davila@epn.edu.ec

2. Docente - Investigador de la Escuela Politécnica Nacional (Ecuador), Economista, MSc. E-mail: napoleon.sanchez@epn.edu.ec

3. Docente - Investigador de la Escuela Politécnica Nacional (Ecuador), Ingeniero en Ciencias Económicas y Financieras, MSc. E-mail: luis.roman@epn.edu.ec

Citación sugerida:

Dávila, Á., Sanchez-Choez, N., y Román-Vásquez, J.L. (2017). Pronóstico de índice bursátil ecuatoriano (Ecuindex) mediante redes neuronales autorregresivas. 3C Empresa: investigación y pensamiento crítico, 6(3), 16-32. DOI: 〈http://dx.doi.org/10.17993/3cemp.2017.060331.16-32/>. 


\section{RESUMEN}

El presente trabajo plantea la aplicación de redes neuronales autorregresivas no lineales para el pronóstico del índice bursátil del mercado ecuatoriano de acciones, Ecuindex. Se prueban 45 estructuras de redes tipo NAR; modificando el número de retrasos de la serie de tiempos del índice y el número de neuronas de la capa oculta. En el período de prueba, la mejor red presenta un error MAPE inferior a $0.25 \%$ y un porcentaje de acierto de dirección del cambio superior al 68\%.

\section{ABSTRACT}

This paper proposes the application of nonlinear autoregressive neural networks for the forecast of the stock market index of the Ecuadorian stock market, Ecuindex. Forty-five NAR network structures are tested; modifying the number of lags in the index time series and the number of neurons in the hidden layer. In the test period, the best network has a MAPE error of less than $0.25 \%$ and a percent of success in change direction greater than $68 \%$.

\section{PALABRAS CLAVE}

Ecuindex, redes neuronales autorregresivas, pronósticos, mercado ecuatoriano de acciones, coeficiente de Hurst.

\section{KEY WORDS}

Ecuindex, autoregressive neural networks, forecasts, Ecuadorian stock market, Hurst coefficient. 


\section{INTRODUCCIÓN}

En el Ecuador, el Mercado bursátil está atendido por las Bolsas de Valores de Guayaquil (BVG) y Quito (BVQ) (Aguirre Gómez \& Romero Morán, 2016). La BVQ ha elaborado, desde 1993, el índice bursátil denominado Ecuindex, el cual "está compuesto por una canasta de los diez emisores más representativos del último semestre respecto a su capitalización bursátil, número de transacciones y monto efectivo negociado (Bolsa de Valores de Quito, 2017).

Dado que este índice se lo utiliza para observar las variaciones diarias de las acciones que se cotizan a nivel nacional (Bolsa de Valores de Quito, 2017), el pronóstico del mismo es de gran interés para inversionistas potenciales y actuales a fin de tomar decisiones de compra o venta que les generen beneficios.

Detectar las tendencias de los datos bursátiles es una tarea difícil, ya que tienen comportamientos complejos, no lineales, dinámicos y caóticos. Los métodos estadísticos tradicionales en los cuales los pronosticadores confiaron en años recientes, parecen fallar en capturar la relación entre las variables del mercado (Dunis, Laws, \& Karathanasopoulos, 2011).

Por las razones expuestas en el párrafo anterior y en vista de que las redes neuronales artificiales son usadas por su habilidad para aprender las complejidades de sistemas no lineales (Benedetti, Baigún, \& Yanguas, 2011), se plantea en este estudio la aplicación de estas redes para pronosticar el Ecuindex. En los siguientes párrafos se exponen estudios previos relacionados con la aplicación de redes neuronales a pronósticos en mercados bursátiles.

Hadavandi, Shavandi, \& Ghanbari, (2010) aplicaron un modelo híbrido basado en lógica difusa y redes neuronales al pronóstico de acciones del sector de tecnología y de aerolíneas en Irán y obtuvieron mejores resultados en términos de error porcentual absoluto medio (MAPE) al comparar el modelo híbrido con modelos basados en redes neuronales solamente.

Guresen, Kayakutlu, \& Daim, (2011) compararon una red tipo perceptrón, una red neuronal dinámica y una red neuronal híbrida basada en un modelo GARCH (Heterocedasticidad condicional autorregresiva) para pronosticar los valores de cierre del índice NASDAQ. La comparación fue realizada en términos del error cuadrático medio (MSE) y desviación media absoluta (MAD). El tercer modelo presenta mejores resultados.

Wang, Wang, Zhang, \& Guo, (2011) compararon una red neuronal tipo wavelet con propagación hacia atrás con una red neuronal simple con propagación hacia atrás. Las redes se aplicaron para predecir el índice compuesto de Shanghái con datos mensuales de cierres desde enero de 1993 hasta diciembre de 2009. La red tipo wavelet presentó mejor desempeño.

Dunis, Laws, \& Karathanasopoulos, (2011) usaron una combinación de un modelo media móvil autorregresiva (ARMA) y una red neuronal. El modelo combinado se usó para 
pronosticar el índice bursátil de Atenas (ASE) durante el período 2001 - 2008, y superó a otros seis modelos basados únicamente en redes neuronales en términos de retorno anualizado en una simulación de inversión.

Wanjawa \& Muchemi, (2014) aplicaron una red neuronal multicapa con alimentación hacia adelante al pronóstico de mercados bursátiles (Nairobi Securities Exchange y New York Stock Exchange). Los resultados de predicción presentaron error porcentual absoluto medio (MAPE) entre $0.71 \%$ y $2.77 \%$.

En un contexto más cercano, (Benedetti, Baigún, \& Yanguas, 2011) aplicaron redes neuronales para predecir el índice bursátil Merval en Argentina y encontraron que son un buen predictor. En todos los modelos desarrollados la estrategia de compra/venta con redes neuronales genera ganancias que superan ampliamente a la estrategia de comprar y mantener.

En Colombia, Villada, Muñoz, \& García, (2012) probaron varias configuraciones de redes neuronales aplicadas al pronóstico del precio de las acciones de Ecopetrol y Preferencial Bancolombia, empresas que cotizan en Bolsa de Colombia y de Nueva York. "Los resultados muestran un buen comportamiento de las redes neuronales con bajos errores en su desempeño tanto en aprendizaje como en predicción" (Villada, Muñoz, \& García, 2012, pág. 11). Se utilizó una serie de 6 meses: cinco para entrenamiento y uno para evaluación de la capacidad predictiva. Se utiliza RMSE (Raíz del error medio cuadrático) y el MAPE como indicadores del error para la comparación entre las redes desarrolladas.

Finalmente, la presente investigación se considera un aporte investigativo, ya que no se encontraron estudios similares sobre pronóstico del Ecuindex, al revisar las siguientes bases de datos: Scopus, Elsevier, Ebsco, e-libro, ProQuest, Springer, arXiv, Google Académico.

\section{REDES NEURONALES ARTIFICIALES}

Las redes neuronales son útiles para realizar un mapeo entrada-salida cuando la relación exacta entre entrada-salida es muy compleja para ser determinada matemáticamente. Dada su habilidad para aprender mapeos complejos, han sido usadas para modelar relaciones económicas no lineales. Presentando un conjunto de datos de pares entradasalida iterativamente, una red neuronal puede ser entrenada para determinar un conjunto de pesos que pueden aproximar el mapeo (Kamruzzaman \& Sarker, 2003).

La red neuronal multicapa de propagación hacia adelante (Figura 2) es una de las arquitecturas más usadas. Consiste de una capa de entrada, una capa de salida y una o más capas intermedias llamadas capas ocultas. Todos los nodos en cada capa están conectados a cada nodo en la capa superior mediante fuerzas de interconexión llamadas pesos. $x_{i}$ son las entradas, $w$ son los pesos, $y_{k}$ son las salidas producidas por la red. Todos los pesos interconectados entre capas son inicializados a pequeños valores aleatorios al inicio. 
Durante el entrenamiento las entradas son presentadas en la capa de entrada y la salida objetivo asociada es presentada en la capa de salida.

Un algoritmo de entrenamiento es utilizado para obtener un conjunto de pesos que minimiza la diferencia entre la salida objetivo y la salida real producida por la red. (Kamruzzaman \& Sarker, 2003), citado en (Dávila \& Herrera, 2015).

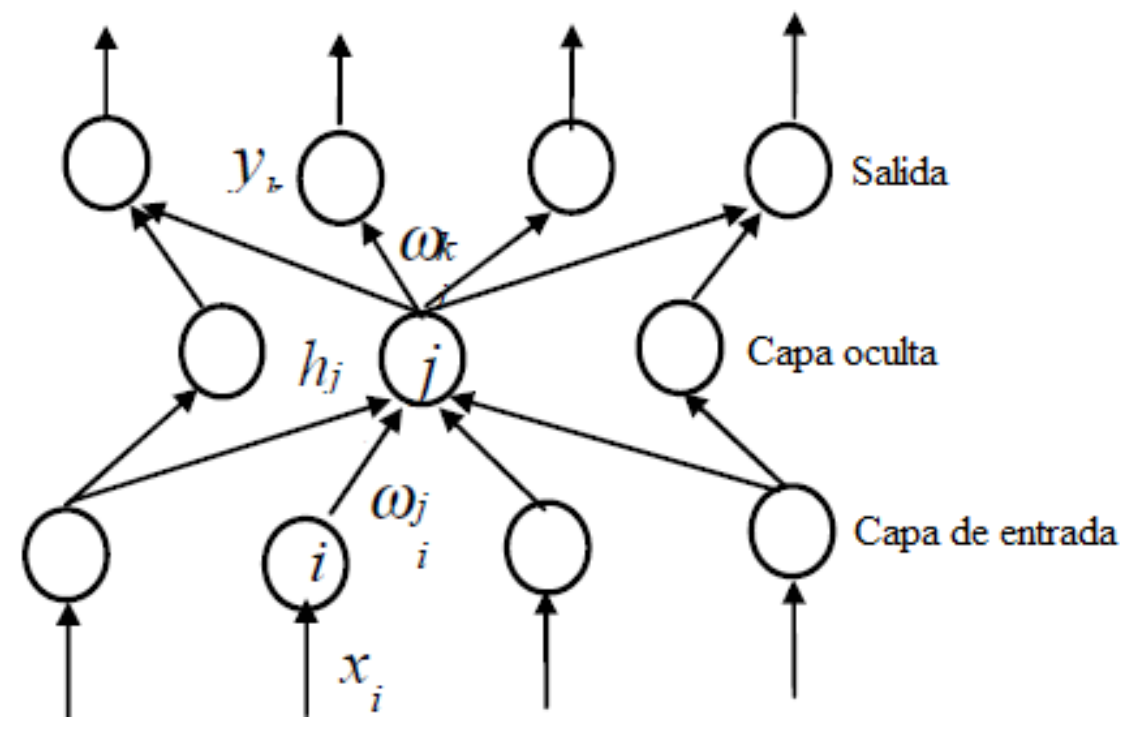

Figura 2. Estructura de una red neuronal multicapa con propagación hacia adelante.

Adaptado de: Kamruzzaman \& Sarker, (2003, pág. 5).

El algoritmo que tradicionalmente se ha empleado para el entrenamiento es el de retropropagación. Se calcula primero la salida, y luego se va calculando capa por capa, hacia atrás, el error de forma cuadrática, para obtener el valor con que los pesos deben ser ajustados (Barragán, 2004).

\section{PRONÓSTICO DEL ECUINDEX MEDIANTE REDES NEURONALES AUTORREGRESIVAS NO LINEALES}

Para este estudio se plantea abordar el problema de pronóstico del Ecuindex basado en datos históricos (retornos) del propio índice, de forma similar al estudio del precio de las acciones en Colombia (Villada, Muñoz, \& García, 2012).

\subsection{ARQUITECTURA RED NEURONAL}

Existen diferentes arquitecturas de redes neuronales, y aunque la más utilizada es la del tipo propagación hacia adelante (Villada, Muñoz, \& García, 2012), se plantea utilizar una arquitectura de tipo autorregresiva no lineal (NAR). Se escoge esta arquitectura de red, ya que su estructura permite colocar a la salida el valor pronosticado de una variable (en este 
caso el pronóstico del Ecuindex) en función de valores anteriores (retrasos) de la misma variable. Para la ejecución de los modelos se utiliza el programa Matlab R2017a, el cual ofrece una arquitectura prediseñada de red NAR, como parte un conjunto de herramientas de redes neuronales para aplicación en pronóstico de series de tiempo. La Figura 3 muestra la arquitectura del tipo de red utilizada.

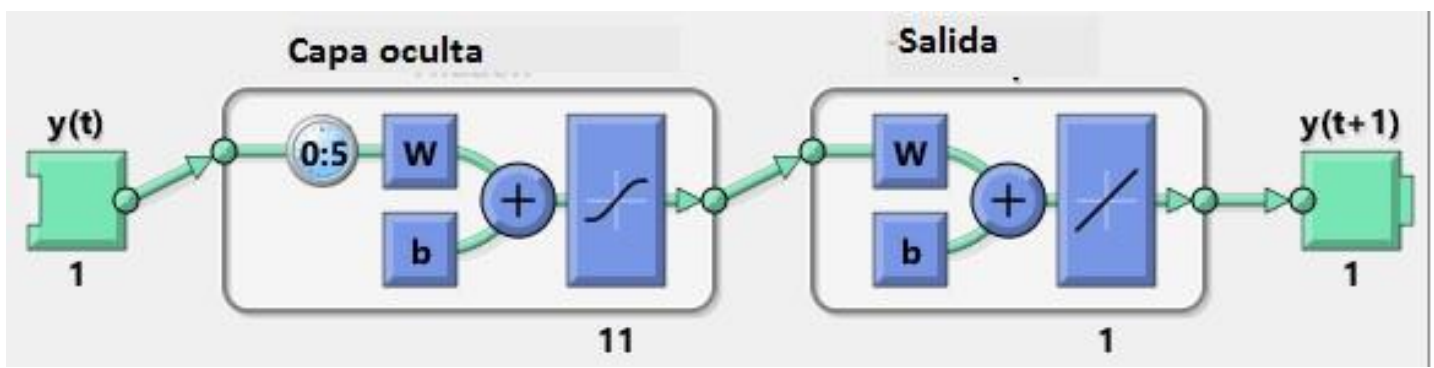

Figura 3. Arquitectura de red NAR (autorregresiva no lineal).

Fuente: elaboración propia, generado con el software Matlab.

\subsection{INFORMACIÓN HISTÓRICA DEL ECUINDEX}

Al momento del estudio se dispone de la información del Ecuindex como una serie de tiempo de valores diarios (de lunes a viernes) desde sus inicios el 2/ago./1993 hasta el $2 /$ mar./2017. Sin embargo, se considera inicialmente la serie únicamente desde el lunes 11/jun./2001, debido a que el viernes 8/jun./2001 fue el último plazo para cambiar la moneda local anterior (Sucres) por dólares americanos (Cabezas, Egüez, Hidalgo, \& Pazmiño, 2017), que es desde entonces la moneda vigente. La información fue provista directamente por un funcionario autorizado de la Bolsa de Valores de Quito (BVQ). La serie se muestra en la Figura 4.

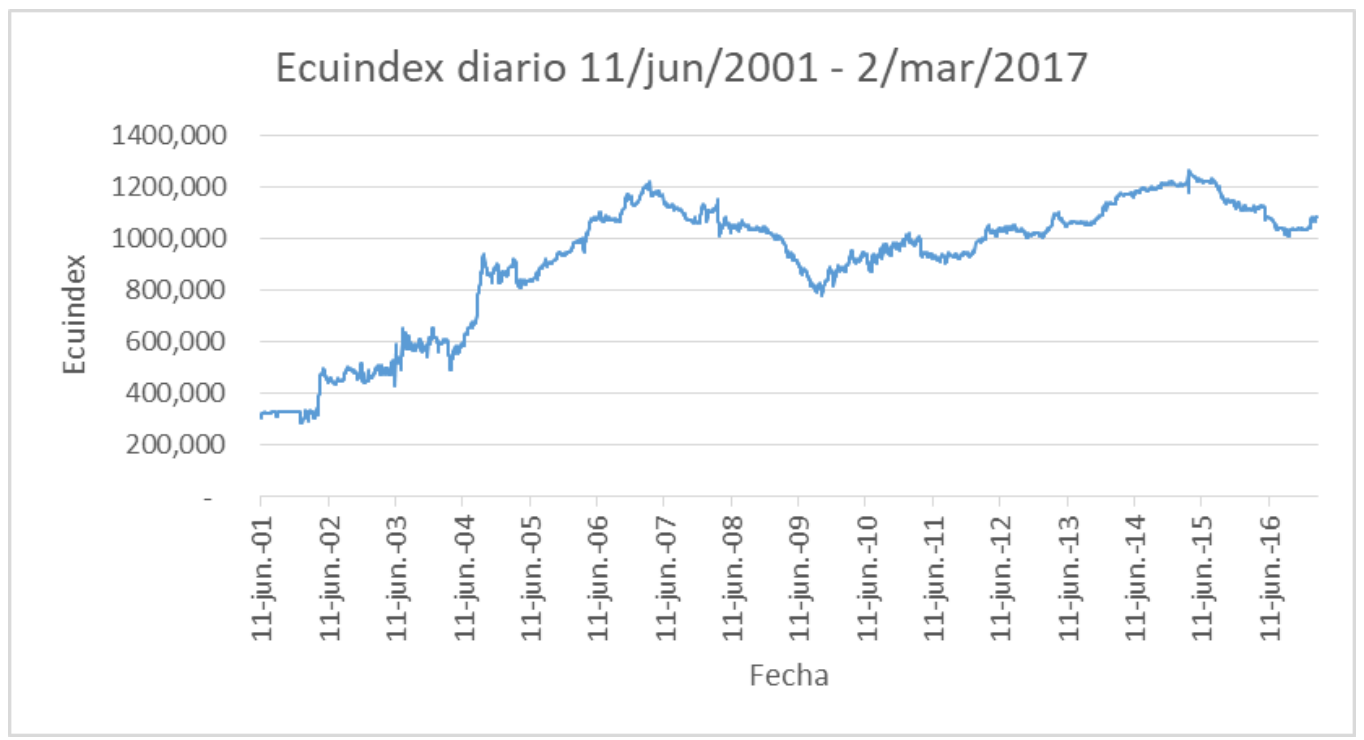

Figura 4. Serie de valores diarios del Ecuindex, 11/jun/2001 - 2/mar/2017. Fuente: elaboración propia, en base a datos de la BVQ. 


\subsection{PRE-PROCESAMIENTO DE LA INFORMACIÓN}

Usualmente existe una fuerte correlación entre los valores actuales y previos de los valores de series financieras, con lo cual el valor más probable del valor en el siguiente período es igual al valor en su anterior período (Kondratenko \& Kuperin, 2003).

Por estas razones, se opta (como alternativa de pre-procesamiento) por utilizar los retornos de los valores del indicador, como se define en la ecuación (1) (Dunis, Laws, \& Sermpinis, 2009):

$$
R_{t}=\left(\frac{I_{t}}{I_{t-1}}\right)-1
$$

Donde:

$R_{t}=$ la tasa de retorno en el tiempo $t ;$

$I_{t}=$ nivel del indicador en el tiempo $t$;

Los retornos de precios también fueron utilizados en estudios anteriores de pronósticos como el de (Dunis, Laws, \& Sermpinis, 2009) y Gómez (2007).

Los retornos del Ecuindex desde 11/jun/2001 hasta el 2/mar/2017 se muestran en la Figura 5.

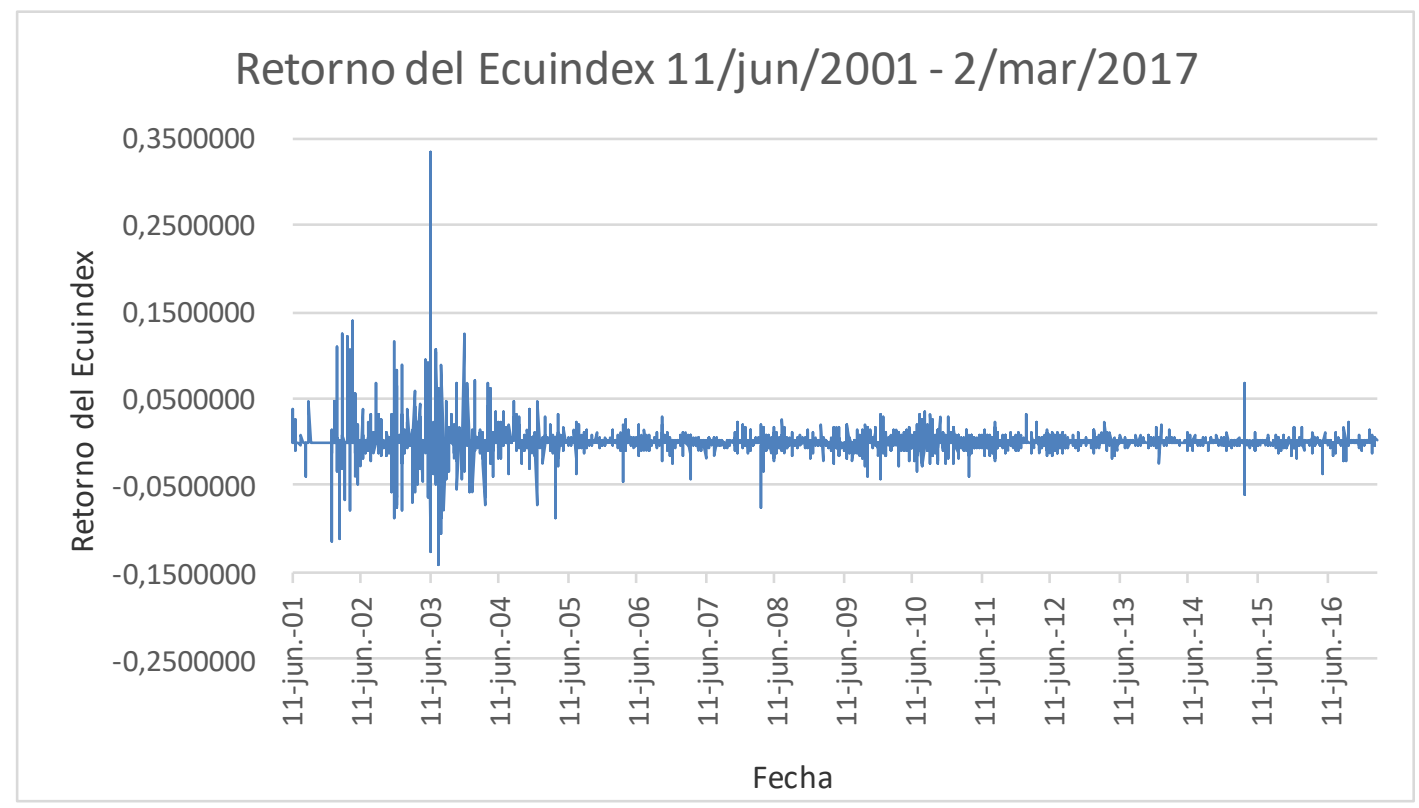

Figura 5. Serie de retornos del Ecuindex, 11/jun/2001 - 2/mar/2017.

Fuente: elaboración propia. 
A fin de seleccionar la ventana de datos a utilizar efectivamente para el pronóstico mediante redes neuronales, se utiliza el exponente de Hurst. El exponente de Hurst es un valor numérico que hace posible determinar la auto-correlación en una serie de datos (Quintero \& Ruiz, 2011), lo cual nos indica en algún sentido la posibilidad de realizar pronósticos (Kondratenko \& Kuperin, 2003). El análisis mediante el coeficiente de Hurst se utilizó también en estudios previos similares de pronósticos de precios de mercado de divisas como el de (Kondratenko \& Kuperin, 2003) y el de (Yao \& Tan, 2000).

Se contemplan tres casos para el coeficiente de Hurst $(\mathrm{H})$. Primero, las series con $\mathrm{H}=0.5$ son puramente aleatorias (ruido blanco); $\mathrm{H}=0.5$ implica un proceso completamente aleatorio e independiente. Segundo, $0.5<H \leq 1$ implica series de tiempo que muestran procesos persistentes o correlacionados ( $\mathrm{H}=1$ indicaría un comportamiento determinístico).

Tercero, $0 \leq H<0.5$ corresponde a un comportamiento de anti-persistencia o anticorrelacional en la serie de tiempo (Quintero \& Ruiz, 2011).

Es deseable entonces para favorecer la posibilidad de realizar pronósticos, que la serie de tiempo tenga un coeficiente $\mathrm{H}$ distinto de 0.5 .

La Figura 6 muestra la variación del coeficiente de Hurst con la fecha de inicio de la serie de datos. Si se tomaran los datos desde el 11/jun/2001 se tendría un valor $\mathrm{H}$ de 0,57. Se busca un valor alejado de 0.5 (lo cual indicaría correlación entre los datos de la serie) en los últimos años y con tal criterio se selecciona la fecha 2/abr/2015, el cual presenta un valor $\mathrm{H}$ de 0.61 . Es decir, que la serie seleccionada para el pronóstico es la serie de retornos del Ecuindex comprendida entre el 2/abr/2015 y el 2/mar/2017.

No se presentan detalles del cálculo del coeficiente de Hurst, el cual se realizó con el software NumXL (v. 1.64).

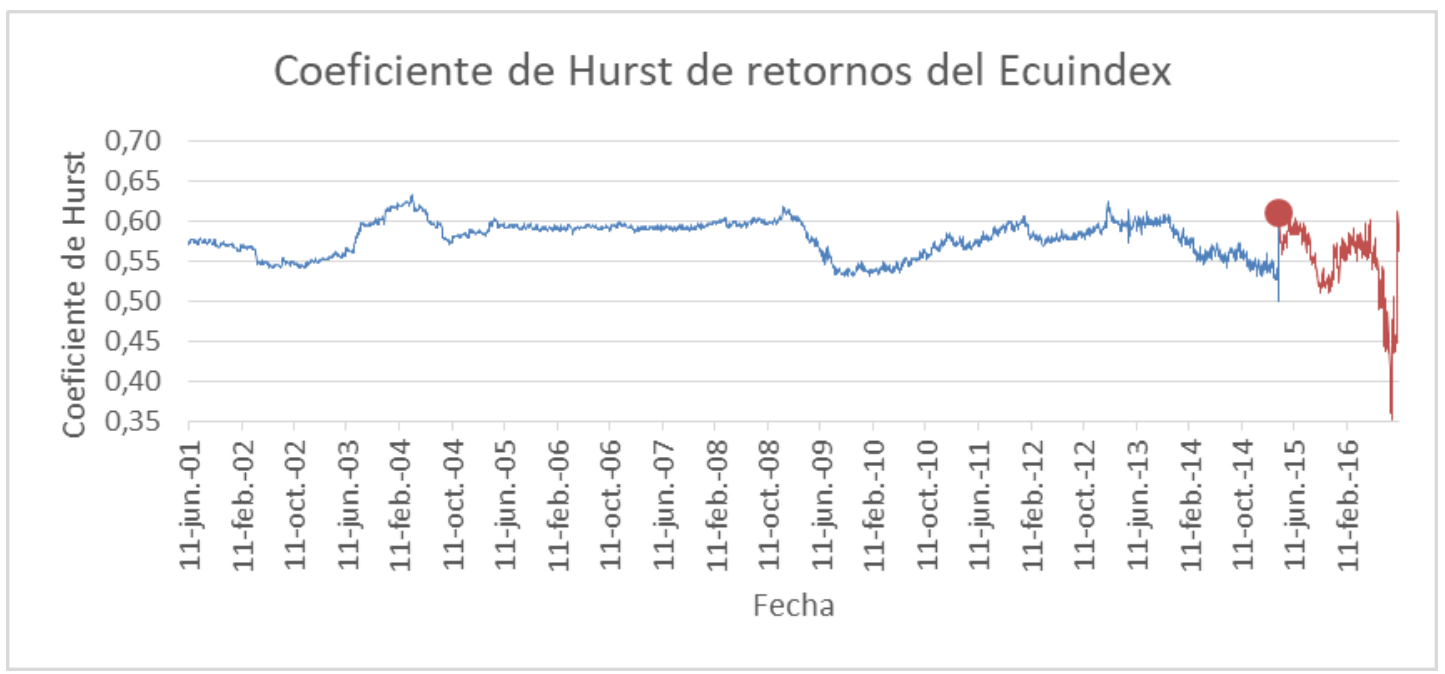

Figura 6. Coeficiente de Hurst de los retornos del Ecuindex.

Fuente: elaboración propia. 


\subsection{SERIE DE DATOS SELECCIONADA}

La serie de datos seleccionada, en base al criterio del coeficiente de Hurst, es la de 478 valores de retornos del Ecuindex comprendida entre el 2/abr/2015 y el 2/mar/2017.

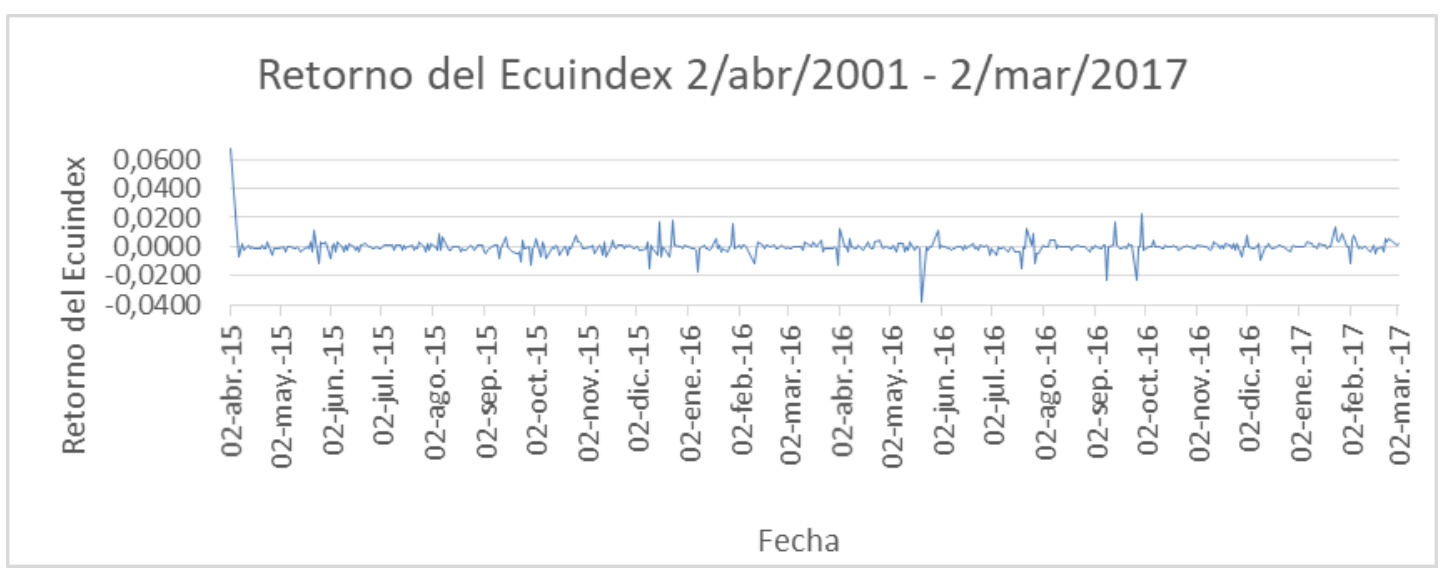

Figura 7. Serie de retornos del Ecuindex, 2/abr/2001 - 2/mar/2017.

Fuente: elaboración propia.

A continuación, se presenta un breve resumen de análisis estadístico de la serie de datos seleccionada.

Tabla 1. Análisis Estadístico de la serie de retornos del Ecuindex.

\begin{tabular}{|l|r|}
\hline \multicolumn{2}{|c|}{ Retornos del Ecuindex } \\
\hline Media & -0.000154 \\
\hline Error típico & 0.00024849 \\
\hline Mediana & 0 \\
\hline Moda & 0 \\
\hline Desviación estándar & 0.00543282 \\
\hline Varianza de la muestra & $2.9516 \mathrm{E}-05$ \\
\hline Curtosis & 57.4248756 \\
\hline $\begin{array}{l}\text { Coeficiente de } \\
\text { asimetría }\end{array}$ & 3.32327329 \\
\hline Rango & 0.10568984 \\
\hline Mínimo & -0.0380899 \\
\hline Máximo & 0.06759991 \\
\hline Suma & -0.0736057 \\
\hline Cuenta & 478 \\
\hline
\end{tabular}

Fuente: elaboración propia. 


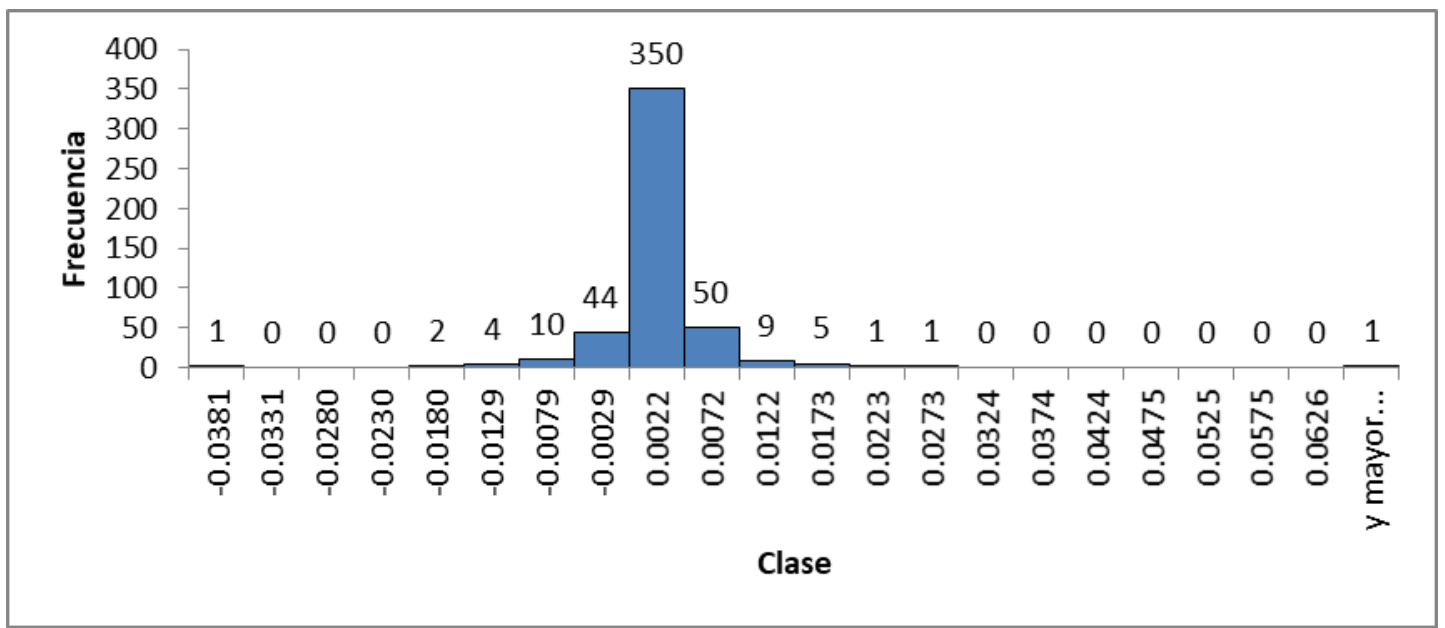

Figura 8. Histograma retornos del Ecuindex, 2/abr/2001 - 2/mar/2017.

Fuente: elaboración propia.

\subsection{TEST DE NORMALIDAD}

Adicionalmente a la evaluación de la persistencia de la serie de datos (mediante $\mathrm{H}$ ), es necesario determinar si la serie tiene una distribución normal (Gaussiana). Mientras $\mathrm{H}$ es más cercano a 1 y mientras más difiera la distribución de la serie de la de una distribución normal, mayor es la probabilidad de que las series puedan ser pronosticadas con calidad (Kondratenko \& Kuperin, 2003). Para evaluar la normalidad de la serie, se utiliza el test de Jarque Bera, como se realizó en el estudio de (Dunis, Laws, \& Sermpinis, 2009). En este estudio no se presentan los detalles de cálculo del test de normalidad, el cual se efectúa sobre la serie seleccionada de retornos de precios mediante el software NumXI, para un nivel de significancia de $1 \%$.

Los resultados del test muestran (Tabla 2) que la serie es NO normal, lo cual es favorable para realizar el pronóstico.

Tabla 2. Resultados del test de normalidad (Jarque Bera).

\begin{tabular}{|r|c|c|c|c|c|}
\hline $\begin{array}{r}\text { Prueba de } \\
\text { Normalidad }\end{array}$ & Puntuación & C.V. & Valor $\mathbf{P}$ & ¿Normalidad? & $\mathbf{1 . 0 \%}$ \\
\hline Jarque-Bera & 65160.66 & 9.21 & $0.0 \%$ & FALSO & Significancia \\
\hline
\end{tabular}

Fuente: elaboración propia.

\subsection{TEST DE ESTACIONARIDAD}

Las series de tiempo financieras son intrínsecamente no estacionarias (Kondratenko \& Kuperin, 2003). La mayoría de métodos estadísticos de pronóstico están basados en el supuesto de que las series de tiempo pueden ser representadas por series aproximadamente estacionarias ("estacionarizadas") a través de transformaciones matemáticas (Nau, 2005).En nuestro caso, la transformación que utilizamos es la de usar una serie de retornos del Ecuindex (sección 0 ) en lugar de la serie original del índice. 
Para evaluar la estacionaridad de la serie de retornos de precio escogida, se utiliza el test de ADF (Augmented Dickey Fuller), el cual fue utilizado en un estudio anterior de pronósticos realizado por (Dunis, Laws, \& Sermpinis, 2009). No se detallan en este estudio los detalles de cálculo del test ADF; para el cual se utiliza NumXI. Los resultados del test ADF se muestran en la Tabla 3, e indican que la serie de datos de retornos de precio efectivamente es estacionaria con un nivel de significancia del $1 \%$.

Tabla 3. Resultados del test de estacionaridad (Augmented Dickey Fuller).

\begin{tabular}{|c|c|c|c|c|c|}
\hline Prueba de Estacionaridad & & & & & \\
\hline Prueba & Puntuación & Valor $\mathbf{P}$ & C.V. & Estacionaridad? & $1.0 \%$ \\
\hline ADF & & & & & Significancia \\
\hline No Const & -14.9 & $0.1 \%$ & -2.6 & VERDADERO & \\
\hline Constante-Solamente & -15.0 & $0.1 \%$ & -3.5 & VERDADERO & \\
\hline Constante + Tendencia & -15.1 & $0.0 \%$ & -2.3 & VERDADERO & \\
\hline Const+Tend+Tend^2 & -15.2 & $0.0 \%$ & -2.3 & VERDADERO & \\
\hline
\end{tabular}

Fuente: elaboración propia.

\subsection{DIVISIÓN DE LOS DATOS}

Cuando se entrenan redes multicapa, es necesario dividir los datos en tres subconjuntos. El primer subconjunto es el de entrenamiento, usado para calcular el gradiente y actualizar los pesos y offsets de la red. El segundo subconjunto es el de validación. El error (medida de la diferencia entre la salida deseada y la salida generada por la red) es monitoreado durante el proceso de entrenamiento. El conjunto de pruebas (tercer conjunto de datos) no es usado durante el entrenamiento, pero es usado para comparar diferentes redes.

Se propone la estructura 70\%, 20\%, 10\% como se realizó en (Han, 2004).

Tabla 4. División de datos.

División de 478 datos: 2/abr/2015 - 2/mar/2017

\begin{tabular}{|c|c|c|}
\hline Entrenamiento: 70\%, 336 datos & Validación: $20 \%, 95$ datos & Prueba: $10 \%$, 47 datos \\
\hline 2/abr/2015 - 3/ago/2016 & 4/ago/2016-21/dic/2016 & 22/dic/2016-2/mar/2017 \\
\hline
\end{tabular}

Fuente: elaboración propia.

\subsection{ALGORITMO DE ENTRENAMIENTO}

Las redes multicapa con retropropagación (para el entrenamiento) son las más utilizadas en pronóstico de series de tiempo financieras (Kaastra \& Boyd, 1996), por lo cual se propone utilizar el algoritmo Levenberg-Marquardt backpropagation.

\subsection{ENTRENAMIENTO DE LAS REDES NEURONALES}

Decidir sobre una red neuronal apropiada involucra mucho ensayo y error. Determinar el número óptimo de neuronas ocultas es un factor crucial para un buen diseño de una red, ya que las neuronas ocultas proveen habilidad para generalizar, sin embargo, en la mayoría de 
situaciones no hay una manera de determinar el mejor número de neuronas sin entrenar varias redes (Dunis, Laws, \& Naim, 2003). Para este estudio se evalúan combinaciones de retrasos desde 2 hasta 6 de la serie del Ecuindex como entradas de la red (en el estudio de (Villada, Muñoz, \& García, 2012) se probaron hasta 5 retrasos). Para el número de neuronas de la capa oculta de la red se propone probar con 4 hasta 12 (en el estudio de (Villada, Muñoz, \& García, 2012) se probaron hasta 10 neuronas). Resultan 45 redes neuronales a entrenar y evaluar, nombradas de la " 1 " a la " 45 ". Las 45 estructuras se resumen en la Tabla $5 ; r$ representa el orden de los retrasos ( 2 a 6 ) y $n n$ el número de neuronas en la capa oculta.

Tabla 5. Redes entrenadas y evaluadas.

\begin{tabular}{|r|r|r|}
\hline Red & $\mathbf{r}$ & nn \\
\hline 1 & 2 & 4 \\
\hline 2 & 2 & 5 \\
\hline 3 & 2 & 6 \\
\hline 4 & 2 & 7 \\
\hline 5 & 2 & 8 \\
\hline 6 & 2 & 9 \\
\hline 7 & 2 & 10 \\
\hline 8 & 2 & 11 \\
\hline 9 & 2 & 12 \\
\hline
\end{tabular}

\begin{tabular}{|r|r|r|}
\hline Red & r & nn \\
\hline 10 & 3 & 4 \\
\hline 11 & 3 & 5 \\
\hline 12 & 3 & 6 \\
\hline 13 & 3 & 7 \\
\hline 14 & 3 & 8 \\
\hline 15 & 3 & 9 \\
\hline 16 & 3 & 10 \\
\hline 17 & 3 & 11 \\
\hline 18 & 3 & 12 \\
\hline
\end{tabular}

\begin{tabular}{|r|r|r|}
\hline Red & \multicolumn{1}{r|}{} & nn \\
\hline 19 & 4 & 4 \\
\hline 20 & 4 & 5 \\
\hline 21 & 4 & 6 \\
\hline 22 & 4 & 7 \\
\hline 23 & 4 & 8 \\
\hline 24 & 4 & 9 \\
\hline 25 & 4 & 10 \\
\hline 26 & 4 & 11 \\
\hline 27 & 4 & 12 \\
\hline
\end{tabular}

\begin{tabular}{|r|r|r|}
\hline Red & $\mathbf{r}$ & nn \\
\hline 28 & 5 & 4 \\
\hline 29 & 5 & 5 \\
\hline 30 & 5 & 6 \\
\hline 31 & 5 & 7 \\
\hline 32 & 5 & 8 \\
\hline 33 & 5 & 9 \\
\hline 34 & 5 & 10 \\
\hline 35 & 5 & 11 \\
\hline 36 & 5 & 12 \\
\hline
\end{tabular}

\begin{tabular}{|r|r|r|}
\hline Red & $\mathbf{r}$ & nn \\
\hline 37 & 6 & 4 \\
\hline 38 & 6 & 5 \\
\hline 39 & 6 & 6 \\
\hline 40 & 6 & 7 \\
\hline 41 & 6 & 8 \\
\hline 42 & 6 & 9 \\
\hline 43 & 6 & 10 \\
\hline 44 & 6 & 11 \\
\hline 45 & 6 & 12 \\
\hline
\end{tabular}

Fuente: elaboración propia.

\subsection{CRITERIO DE COMPARACIÓN DE LAS REDES}

La medida de calidad de los pronósticos de las diferentes redes se evalúa en función del error: RMSE y el MAPE, calculados mediante las ecuaciones 2 y 3 (Villada, Muñoz, \& García, 2012). Adicionalmente se incluye el indicador de porcentaje de acierto en la predicción de dirección del cambio CDC (correct directional change), definido en la ecuación 4 (Dunis, Laws, \& Naim, 2003). El indicador de acierto en la dirección del cambio es importante porque al predecir correctamente la dirección del índice al alza o a la baja se pueden tomar decisiones acertadas de compra o venta, respectivamente.

$$
\begin{aligned}
& \text { RMSE }=\sqrt{\frac{1}{n} \sum_{t=1}^{n}\left(y_{t}^{\prime}-y_{t}\right)^{2}} \\
& \text { MAPE }=\frac{100}{n} \sum_{t=1}^{n}\left|\frac{y_{t}^{\prime}-y_{t}}{y_{t}}\right|
\end{aligned}
$$




$$
C D C=\frac{100}{N} \sum_{t=1}^{N} D_{t}
$$

Donde, $D_{t}=1$ si $y_{t} \cdot \widehat{y_{t}}>0,0$ si $y_{t}, \widehat{y_{t}} \leq 0$

\section{RESULTADOS}

A continuación, se presentan los resultados del desempeño del pronóstico del Ecuindex de cada una de las 45 redes para el período de prueba de 47 días (22/dic/2016 - 2/mar/2017) definido previamente (sección 0). En la Tabla 6 se muestran los resultados del error RMSE, MAPE, y CDC.

Tabla 6. Desempeño del pronóstico del Ecuindex basado en redes neuronales.

\begin{tabular}{|r|r|r|r|r|c|}
\hline RED & $\mathbf{r}$ & $\mathbf{n n}$ & RMSE & MAPE & CDC \\
\hline 1 & 2 & 4 & 4.37 & $0.265 \%$ & $57.45 \%$ \\
\hline 2 & 2 & 5 & 4.41 & $0.271 \%$ & $57.45 \%$ \\
\hline 3 & 2 & 6 & 4.34 & $0.259 \%$ & $53.19 \%$ \\
\hline 4 & 2 & 7 & 4.39 & $0.267 \%$ & $57.45 \%$ \\
\hline 5 & 2 & 8 & 4.15 & $0.251 \%$ & $59.57 \%$ \\
\hline 6 & 2 & 9 & 4.37 & $0.271 \%$ & $57.45 \%$ \\
\hline 7 & 2 & 10 & 4.36 & $0.267 \%$ & $59.57 \%$ \\
\hline 8 & 2 & 11 & 4.46 & $0.280 \%$ & $59.57 \%$ \\
\hline 9 & 2 & 12 & 4.48 & $0.275 \%$ & $59.57 \%$ \\
\hline 10 & 3 & 4 & 4.36 & $0.267 \%$ & $57.45 \%$ \\
\hline 11 & 3 & 5 & 4.51 & $0.284 \%$ & $55.32 \%$ \\
\hline 12 & 3 & 6 & 4.57 & $0.284 \%$ & $55.32 \%$ \\
\hline 13 & 3 & 7 & 4.42 & $0.275 \%$ & $55.32 \%$ \\
\hline 14 & 3 & 8 & 4.34 & $0.274 \%$ & $53.19 \%$ \\
\hline 15 & 3 & 9 & 4.52 & $0.286 \%$ & $55.32 \%$ \\
\hline 16 & 3 & 10 & 4.55 & $0.286 \%$ & $53.19 \%$ \\
\hline 17 & 3 & 11 & 4.61 & $0.303 \%$ & $55.32 \%$ \\
\hline 18 & 3 & 12 & 4.46 & $0.269 \%$ & $53.19 \%$ \\
\hline 19 & 4 & 4 & 4.47 & $0.283 \%$ & $57.45 \%$ \\
\hline 20 & 4 & 5 & 4.41 & $0.271 \%$ & $55.32 \%$ \\
\hline 21 & 4 & 6 & 4.56 & $0.288 \%$ & $55.32 \%$ \\
\hline 22 & 4 & 7 & 4.83 & $0.290 \%$ & $57.45 \%$ \\
\hline 23 & 4 & 8 & 4.62 & $0.294 \%$ & $53.19 \%$ \\
\hline
\end{tabular}

\begin{tabular}{|r|r|r|r|c|c|}
\hline RED & $\mathbf{r}$ & nn & RMSE & MAPE & CDC \\
\hline 24 & 4 & 9 & 4.38 & $0.273 \%$ & $59.57 \%$ \\
\hline 25 & 4 & 10 & 4.4 & $0.279 \%$ & $55.32 \%$ \\
\hline 26 & 4 & 11 & 4.41 & $0.272 \%$ & $51.06 \%$ \\
\hline 27 & 4 & 12 & 4.38 & $0.268 \%$ & $55.32 \%$ \\
\hline 28 & 5 & 4 & 4.23 & $0.250 \%$ & $57.45 \%$ \\
\hline 29 & 5 & 5 & 4.16 & $0.254 \%$ & $55.32 \%$ \\
\hline 30 & 5 & 6 & 4.53 & $0.287 \%$ & $55.32 \%$ \\
\hline 31 & 5 & 7 & 4.64 & $0.301 \%$ & $55.32 \%$ \\
\hline 32 & 5 & 8 & 4.39 & $0.267 \%$ & $55.32 \%$ \\
\hline 33 & 5 & 9 & 4.33 & $0.260 \%$ & $48.94 \%$ \\
\hline 34 & 5 & 10 & 4.29 & $0.260 \%$ & $59.57 \%$ \\
\hline 35 & 5 & 11 & 4.41 & $0.283 \%$ & $51.06 \%$ \\
\hline 36 & 5 & 12 & 4.65 & $0.307 \%$ & $55.32 \%$ \\
\hline 37 & 6 & 4 & 4.57 & $0.285 \%$ & $55.32 \%$ \\
\hline 38 & 6 & 5 & 4.49 & $0.277 \%$ & $55.32 \%$ \\
\hline 39 & 6 & 6 & 4.35 & $0.259 \%$ & $57.45 \%$ \\
\hline 40 & 6 & 7 & 4.51 & $0.284 \%$ & $53.19 \%$ \\
\hline 41 & 6 & 8 & 4.76 & $0.298 \%$ & $53.19 \%$ \\
\hline 42 & 6 & 9 & 4.24 & $0.254 \%$ & $55.32 \%$ \\
\hline 43 & 6 & 10 & 4.57 & $0.286 \%$ & $57.45 \%$ \\
\hline 44 & 6 & 11 & 4.11 & $0.247 \%$ & $68.09 \%$ \\
\hline 45 & 6 & 12 & 4.42 & $0.281 \%$ & $57.45 \%$ \\
\hline
\end{tabular}

Fuente: elaboración propia. 
Los resultados muestran en general un buen desempeño de las redes neuronales. Con respecto al RMSE, los errores no son comparables directamente entre varios estudios, ya que dependen de la magnitud absoluta de la variable que se esté pronosticando. Para salvar esta dificultad se utiliza el MAPE, que se compara más adelante. Sin embargo, el RMSE se considera aceptable ya que está en promedio en 4.44 (ver Tabla 7) mientras que el promedio del Ecuindex para el período de prueba es de 1059.04, es decir, una desviación relativa del orden de $0.42 \%$.

Por otra parte, en el presente estudio se obtuvieron errores MAPE de entre $0.247 \%$ y 0.307\% (ver Tabla 7), inferiores a los de estudios similares como el de (Wanjawa \& Muchemi, 2014), en el cual se obtuvieron errores porcentuales MAPE de entre $0.71 \%$ y $2.77 \%$.

Adicionalmente, para 44 de 45 redes, se obtuvo un acierto de más del $50 \%$ en el pronóstico de la dirección del cambio del índice (CDC), superando en promedio a un camino aleatorio. Se obtuvieron resultados de CDC de entre $48.94 \%$ y $68.09 \%$ (ver Tabla 7), superiores a los obtenidos en estudios similares como el de (Benedetti, Baigún, \& Yanguas, 2011), que estuvieron entre $35.36 \%$ y $62.50 \%$.

Tabla 7. Resumen del desempeño del pronóstico del Ecuindex basado en redes neuronales.

\begin{tabular}{|c|c|c|c|}
\hline & RMSE & MAPE & \multicolumn{1}{c|}{ CDC } \\
\hline Mínimo & 4.11 & $0.247 \%$ & $48.94 \%$ \\
\hline Máximo & 4.83 & $0.307 \%$ & $68.09 \%$ \\
\hline Promedio & 4.44 & $0.275 \%$ & $56.03 \%$ \\
\hline
\end{tabular}

Fuente: elaboración propia.

Destaca el desempeño de la red " 44 ", con 6 retrasos a la entrada y 11 neuronas en la capa oculta, la cual generó el menor error RMSE (4.11), menor error MAPE (0.247\%), y mayor acierto en la dirección de la predicción (68.09\%).

Finalmente, en la Figura 9 se muestra gráficamente el desempeño del pronóstico de la mejor red ("44") en comparación con el valor real del Ecuindex. 


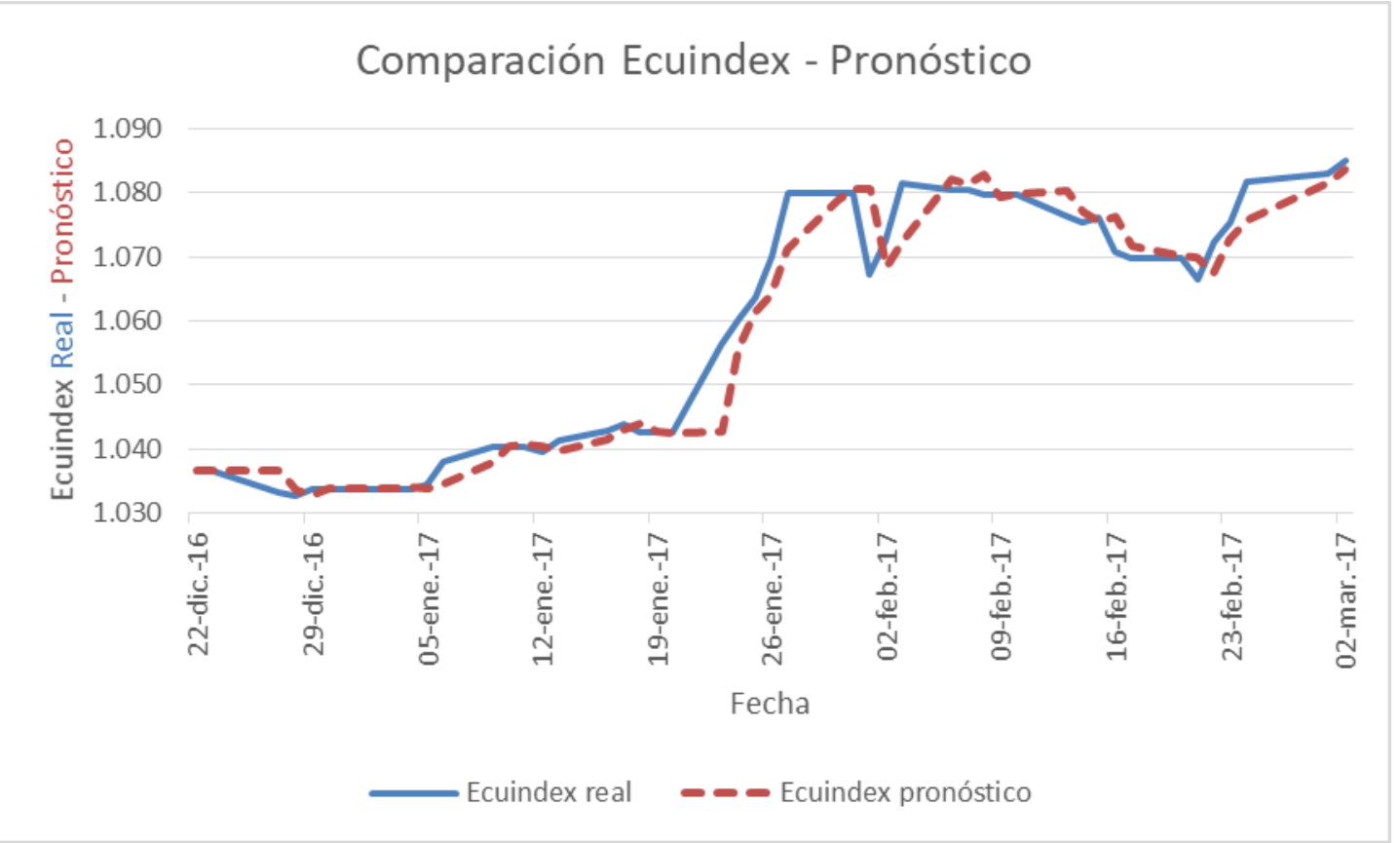

Figura 9. Comparación Ecuindex real - pronóstico.

Fuente: elaboración propia.

\section{CONCLUSIONES}

La aplicación de redes neuronales autorregresivas logró un desempeño exitoso en términos de bajo error (MAPE 0.247\%) y en términos de acierto en la dirección del cambio, que para la mejor red fue de $68 \%$. Un acierto en la predicción del cambio mayor a $50 \%$ en 44 de 45 redes evaluadas, arroja evidencia en contra de la hipótesis de los mercados eficientes, según la cual los precios de las acciones deberían seguir un camino aleatorio. Cabe recalcar que el estudio se hizo para un índice compuesto por una canasta de empresas. En un futuro se podría aplicar las redes neuronales al pronóstico de precios de acciones de empresas representativas en Ecuador.

El uso exitoso de redes neuronales para pronosticar el Ecuindex, abre la posibilidad para probar esta poderosa herramienta en otros mercados bursátiles de economías emergentes similares a la ecuatoriana.

Al no existir una metodología estándar para definir la estructura de las redes neuronales, se hace necesario probar varias redes y seleccionar las de mejor desempeño. En el caso de este estudio aplicado al Ecuindex, la red de mejor desempeño generó un error muy bajo, sin embargo cabe aclarar que no necesariamente las conclusiones para esta estructura de red, son generalizables para otros contextos. En cada contexto habría que ensayar con varias arquitecturas (hacia adelante, autorregresiva, dinámica, etc.) y estructuras de red (variar las entradas y número de neuronas).

Dada la disponibilidad de herramientas de software, la aplicación de redes neuronales resulta relativamente sencilla y permite obtener bajos errores en el pronóstico. 


\section{REFERENCIAS BIBLIOGRÁFICAS}

Aguirre Gómez, L., \& Romero Morán, F. (2016). Manual de Financiamiento para Empresas. Guayaquil: Ediciones Holguín S.A.

Barragán, M. (2004). Cibernética y Control. Quito.

Benedetti, F., Baigún, A., \& Yanguas, M. (2011). Predicciones de Series de Tiempo con Redes Neuronales: Trading usando el Índice Merval. Buenos Aires: Reporte TécnicoUniversidad de San Andrés.

Bolsa de Valores de Quito. (08 de 05 de 2017). Reporte Diario. Obtenido de Bolsa de Valores de Quito: http://www.bolsadequito.info/uploads/estadisticas/cargas/EcuindexIndicadores/ecu-ind.xls

Cabezas, M., Egüez, M., Hidalgo, F., \& Pazmiño, S. (09 de 05 de 2017). La Dolarización en el Ecuador. Obtenido de Banco Central del Ecuador: https://contenido.bce.fin.ec/documentos/PublicacionesNotas/Notas/Dolarizacion/ pdf/Cabezas\%20et\%20al.pdf

Dávila, A., \& Herrera, G. (2015). Estrategia para invertir en el mercado de divisas (Forex) basada en redes neuronales. Revista Politécnica, 35(1).

Dunis, C. L., Laws, J., \& Karathanasopoulos, A. (2011). Modelling and Trading the Greek Stock Market with Mixed Neural Network Models. Applied Financial Economics, 21, 1793-1808.

Dunis, C., Laws, J., \& Naim, P. (2003). Applied quantitative methods for trading and investment. Chichester: John Wiley \& Sons Ltd.

Dunis, C., Laws, J., \& Sermpinis, G. (2009). Modelling and Trading the EUR/USD Exchange Rate at the ECB Fixing. The European Journal of Finance, 16, 541-560. Recuperado el 10 de 5 de 2017, de Liverpool John Moores University: http://www.tandfonline.com/doi/abs/10.1080/13518470903037771

Gómez, G. (2007). Métodos de Predicción para los Mercados de Divisas. Recuperado el 10 de 5 de 2017, de Institute for Research in Technology-Technical Report: www.iit.upcomillas.es/pfc/resumenes/46810bc052797.pdf

Guresen, E., Kayakutlu, G., \& Daim, T. U. (2011). Using artificial neural network models in stock market index prediction. Expert Systems with Applications, 38, 10389-10397. Recuperado el 10 de 5 de 2017

Hadavandi, E., Shavandi, H., \& Ghanbari, A. (2010). Integration of genetic fuzzy systems and artificial neural networks for stock price forecasting. Knowledge-Based Systems, 23, 800-808. 
Han, J. (2004). Forex preditcion using an artificial intelligence system. Recuperado el 10 de 5 de 2017, de Oklahoma State university Library - Tehnical Report: http://digital.library.okstate.edu/etd/umi-okstate-1120.pdf

Kaastra, I., \& Boyd, M. (1996). Desgning a neural network for forecasting financial and economic time series. Neurocomputing, 10, 215-236. Obtenido de Neurocomputing.

Kamruzzaman, J., \& Sarker, R. A. (2003). Comparing ANN Based Models with ARIMA for Prediction of Forex Rates. Asor Bulletin, 22. Obtenido de AUSTRALIAN SOCIETY FOR OPERATIONS RESEARCH INCORPORATED (ASOR).

Kondratenko, V., \& Kuperin, Y. (2003). Using Recurrent Neural Networks to Forecasting of Forex. Recuperado el 10 de 5 de 2017, de Cornell University Library - Technical Report: http://arxiv.org/ftp/cond-mat/papers/0304/0304469.pdf

Nau, B. (2005). Stationarity and differencing. Recuperado el 10 de 5 de 2017, de Forecasting Decision 411: http://people.duke.edu/ rnau/411diff.htm

Quintero, O., \& Ruiz, J. (2011). Estimación del exponente de Hurst y la dimensión fractal de una superficie topográfica a través de la extracción de perfiles. UD y la GEOMÁTICA, 5, 84-91. Obtenido de Universidad Distrital Francisco José de Caldas.

Villada, F., Muñoz, N., \& García, E. (2012). Aplicación de las Redes Neuronales al Pronóstico de Precios en el Mercado de Valores. Información Tecnológica, 23, 11-20.

Wang, J.-Z., Wang, J.-J., Zhang, Z.-G., \& Guo, S.-P. (2011). Forecasting stock indices with back propagation neural network. Expert Systems with Applications, 38, 1434614355.

Wanjawa, B., \& Muchemi, L. (2014). ANN Model to Predict Stock Prices at Stock Exchange Market. Nairobi: University of Nairobi Research Archive.

Yao, J., \& Tan, C. L. (2000). A case study on using neural networks to perform technical forecasting of forex. Neurocomputing, 34, 79-98. 